\title{
Broadband transitions for membrane-based coplanar waveguides on highly-conductive silicon substrates
}

\author{
M. Rohland ${ }^{1,2}$, U. Arz ${ }^{1}$, K. Kuhlmann ${ }^{1}$, and S. Büttgenbach ${ }^{2}$ \\ ${ }^{1}$ Physikalisch-Technische Bundesanstalt, 38116 Braunschweig, Germany \\ ${ }^{2}$ Institut für Mikrotechnik, Langer Kamp 8, 38106 Braunschweig, Germany \\ Correspondence to: M. Rohland (m.rohland@tu-bs.de)
}

\begin{abstract}
We present a transition from a contact structure built on highly-conductive silicon to a coplanar waveguide (CPW) fabricated in membrane technology. Test structures were fabricated and measured. The silicon-to-membrane transition is suitable for on-wafer probing and provides less than $-10 \mathrm{~dB}$ measured reflection in the frequency range from 1 to $110 \mathrm{GHz}$. Measured and calculated values of the propagation constant of the membrane CPW agree well in the entire frequency range.
\end{abstract}

\section{Introduction}

Low-loss, low-dispersion planar transmission line structures are essential for millimeter wave integrated circuits. The advantages of using planar components in microwave circuits are reduced fabrication cost and increased operating bandwidth compared with conventional waveguides like coaxial lines or rectangular waveguides. However, microstrip and coplanar waveguides encounter some problems at millimeter wave frequencies. These are dielectric loss, which increases with frequency, as well as dispersion, substrate moding, and radiation loss, all of which can be directly associated with the air/dielectric interface inherent to all planar transmission lines built in conventional technology.

CPWs on thin insulating carriers are one candidate for high-performance transmission lines without the negative electromagnetic properties mentioned above. Such membrane-based CPWs have been fabricated successfully with the aid of micromachining technologies at the Institute for Microtechnology in Braunschweig, Germany. Due to the very thin carrier membrane beneath the coplanar lines the effective dielectric constant value is close to the ideal value of 1 , leading to lower loss, excellent dispersion properties,

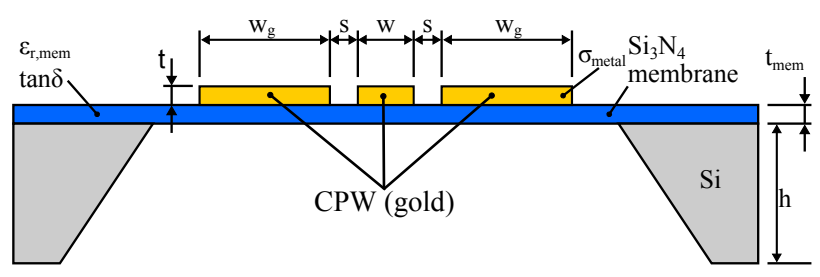

Fig. 1. Cross-sectional view of CPW built in membrane technology.

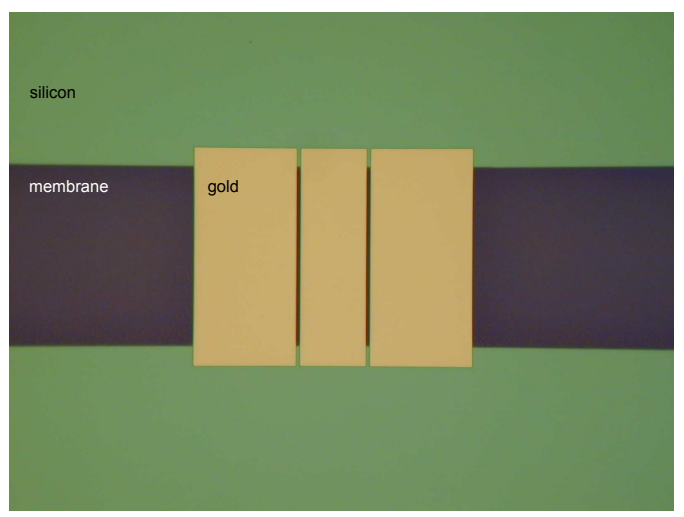

Fig. 2. Top view of $550 \mu \mathrm{m}$ long membrane $\mathrm{CPW}$ test structure.

low radiation, and preventing the excitation of undesired surface modes.

Figure 1 shows a schematic of the cross section of a coplanar line built in membrane technology together with its geometrical and material parameters.

At the time of this investigation, only highly-conductive silicon wafers were available for processing. First, the thin membrane layer is deposited on a highly-conductive silicon wafer. The conducting lines are constructed by sputtering 
and structuring a gold layer on the membrane layer. To selectively remove silicon, wet chemical etching is used. This fabrication step opens the membrane windows which carry the conducting lines.

First prototype test structures included line sections of different lengths and offset shorts. A $550 \mu \mathrm{m}$ long line section is presented in Fig. 2.

In (Rohland et al., 2011) we discussed the advantages of on-wafer membrane-based calibration standards in comparison to standards built in conventional thin-film technology. By means of Monte Carlo simulations we showed that, for coplanar waveguides used as line standards, an up to tenfold reduction in uncertainty can be achieved for certain electromagnetic waveguide properties. The calculations were based on an extension to the analytic model of Heinrich (1993), which was presented in (Arz et al., 2011a).

In (Arz et al., 2011a) we also verified the suitability of the analytic model extension for calculating the broadband line parameters per unit length for CPWs built in membrane technology. The analytic calculations were verified with measurements and full-wave simulations. However, this verification was only possible for the imaginary part of the propagation constant. The absence of a suitable transition from the on-wafer contact pads on the highly-conductive silicon substrate to the membrane-technology-based CPW led to a very high reflection of the signal energy in the silicon part of the interconnect structure. The remaining transmitted signal energy did not allow for a full characterization of the membrane CPW.

In this paper we present a broadband transition with sufficiently low reflection over the entire frequency band. Sect. 2 elaborates on this broadband transition. Both transition design and transition characterization by simulation and measurement are discussed in detail. The membrane CPW with added transitions is investigated by comparing analytical calculations against on-wafer measurements regarding the propagation constant in Sect. 3.

\section{Broadband transition}

The broadband transition has to take into account substrate losses in the highly-conductive silicon. An accurate calculation of the characteristic impedance on silicon is essential for determining line cross-sections that provide a sufficiently low impedance mismatch in the frequency range $1-110 \mathrm{GHz}$. All relevant parts of the new interconnect structure are individually characterized by measurement and calculations.

\subsection{Transition design}

The modified coplanar interconnect structure we introduce in this paper is shown in Fig. 3. Silicon-to-membrane transitions are added to both sides of a coplanar waveguide section fabricated in membrane technology.

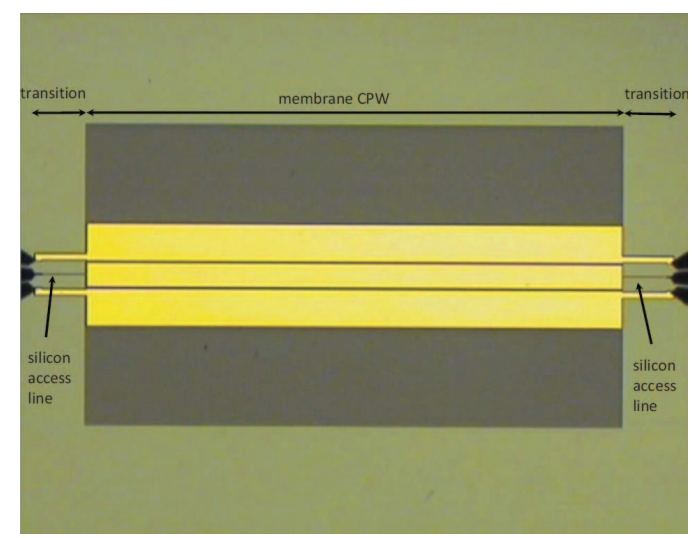

Fig. 3. Membrane CPW with silicon-to-membrane transition.

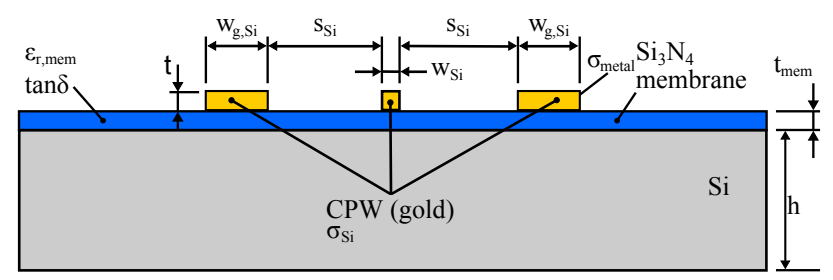

Fig. 4. Cross-sectional view of interconnect segment on silicon (silicon access line).

The silicon-to-membrane transitions are composed of contact pads, which allow for ground-signal-ground (GSG) microwave probing, and a short interconnect segment on silicon. Figure 4 shows a schematic of the cross section of the interconnect segment on silicon together with its geometrical and material parameters.

The pitch size, which is the distance between adjacent probe tips of the GSG probes shown on the right and left side of Fig. 3, is $100 \mu \mathrm{m}$. The values of the cross-sectional parameters of the interconnect structure on silicon are listed in Table 1. In order to achieve a characteristic impedance near the $50 \Omega$ reference impedance of the measurement system, the interconnect segment on the highly-conductive silicon consists of a very narrow signal line (signal line width $w_{\mathrm{Si}}$ ), a wide gap (gap width $s_{\mathrm{Si}}$ ), and relatively narrow ground conductors (ground conductor width $w_{\mathrm{g}, \mathrm{Si}}$ ) on both sides of the signal line.

Regarding the membrane CPW, a wide signal conductor and a very small gap are required to get a characteristic impedance of approximately $50 \Omega$. This is different to CPWs built on bulk substrate materials.

Before fabrication, the electrical properties of the interconnect structure were investigated using a 3-D full-wave time domain electromagnetic field solver (Microwave Studio, 2011). Detailed information will be given in Sect. 2.3. 
Table 1. Geometrical parameters of interconnect segment on silicon.

\begin{tabular}{rr}
\hline parameter & value \\
\hline$w_{\mathrm{g}, \mathrm{Si}}$ & $48 \mu \mathrm{m}$ \\
$w_{\mathrm{Si}}$ & $8 \mu \mathrm{m}$ \\
$s_{\mathrm{Si}}$ & $90 \mu \mathrm{m}$ \\
$t$ & $0.9 \mu \mathrm{m}$ \\
\hline
\end{tabular}

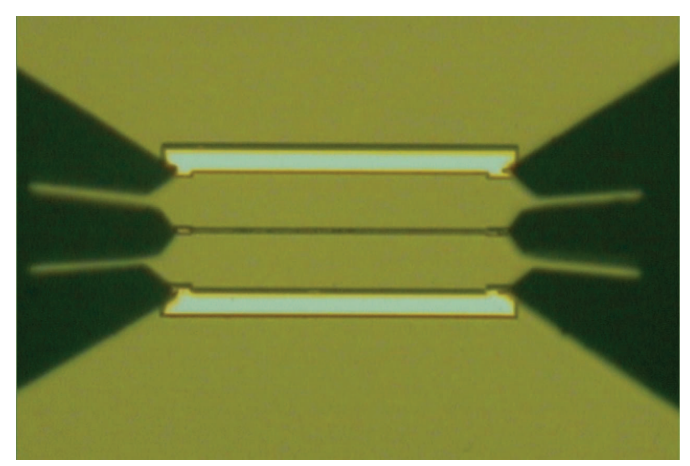

Fig. 5. Interconnect section on silicon.

\subsection{Characterization of access lines on silicon}

To investigate the electromagnetic propagation properties of the interconnect segment on silicon, the so-called access line, we measured test structures fabricated on the same silicon wafer as the membrane CPWs. The top view of a $0.5 \mathrm{~mm}$ long access line test structure is shown in Fig. 5. These test structures are lines with the same cross section as the access lines (see Table 1). Their lengths vary from $0.5 \mathrm{~mm}$ up to more than $20 \mathrm{~mm}$ and are given in Table 2. Together with an offset short they form a set of standards suitable for multiline thru-reflect-line (TRL) calibration (Marks, 1991), which allows for an accurate extraction of the frequency-dependent propagation constant $\gamma$.

Two steps were necessary to determine the characteristic impedance $Z_{0}$ of the access lines on silicon. First, we performed a $50 \Omega$ multiline TRL reference calibration in coplanar lines fabricated on a semiinsulating gallium-arsenide substrate, and moved the calibration reference plane back to the probe tips. Then the characteristic impedance of the access lines was determined by performing a second-tier TRL calibration in the access line test structures on the silicon substrate. To that end, the calibration comparison method (Arz et al., 2000) was employed. This method is designed to be insensitive to large contact-pad capacitance.

To verify the experimental data we used the quasianalytical method of (Grotelüschen et al., 1993). Based on the assumption that the skin effect in the conductive substrate affects only the calculation of the impedance parameters, the procedure of (Grotelüschen et al., 1993) derives the
Table 2. Lengths of access line test structures.

\begin{tabular}{rr}
\hline no. & line length \\
\hline 1 & $0.5 \mathrm{~mm}$ \\
2 & $2.632 \mathrm{~mm}$ \\
3 & $3.69 \mathrm{~mm}$ \\
4 & $7.072 \mathrm{~mm}$ \\
5 & $20.19 \mathrm{~mm}$ \\
\hline
\end{tabular}
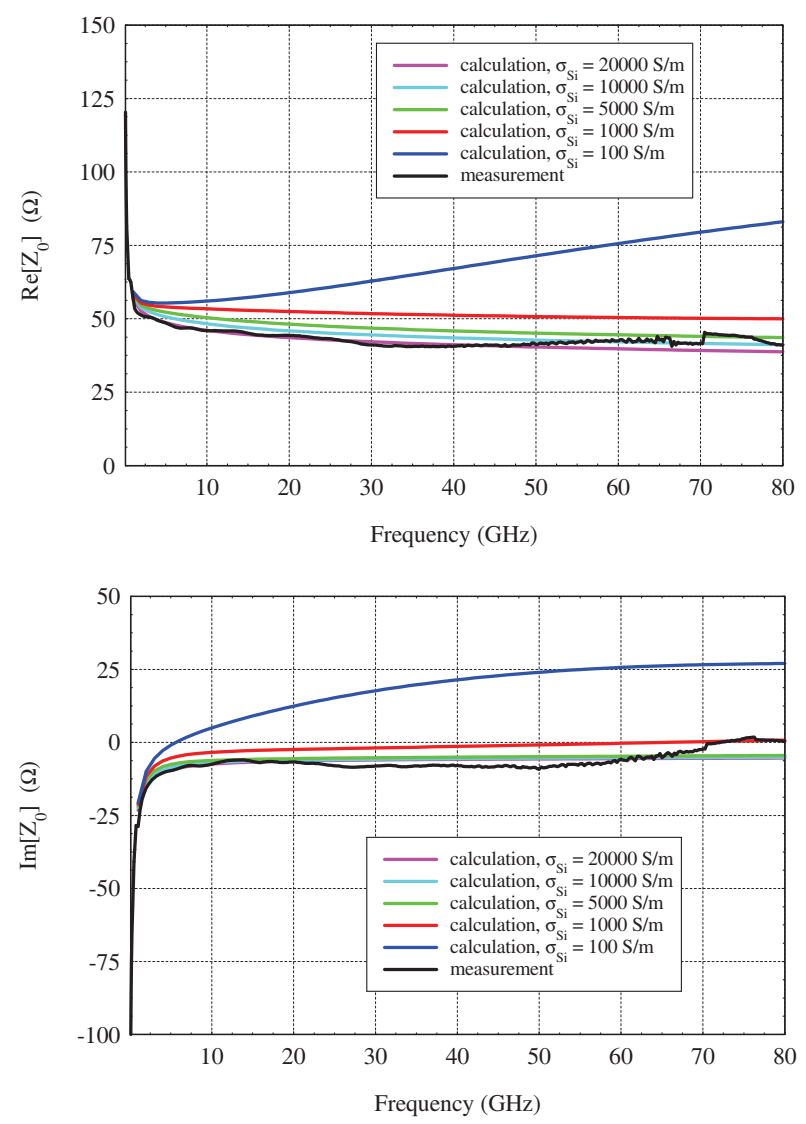

Fig. 6. Real and imaginary part of $Z_{0}$ of silicon access lines.

frequency-dependent line parameters of transmission-line systems on conductive substrates from their cross-sectional data. The skin effect in the signal and ground conductors is neglected.

Figure 6 shows the real and imaginary part of the characteristic impedance $Z_{0}$ of the access lines on silicon. Not knowing the substrate conductivity $\sigma_{\mathrm{Si}}$ with sufficient accuracy in advance, we performed the calculations using different values for that parameter. The manufacturer specified a wafer conductivity above $5000 \mathrm{~S} \mathrm{~m}^{-1}$. Thus, we chose additional values in the calculations to demonstrate the influence of the substrate conductivity on the silicon access line parameters. The measured characteristic impedance agrees well with the quasi-analytical calculations of (Grotelüschen et al., 


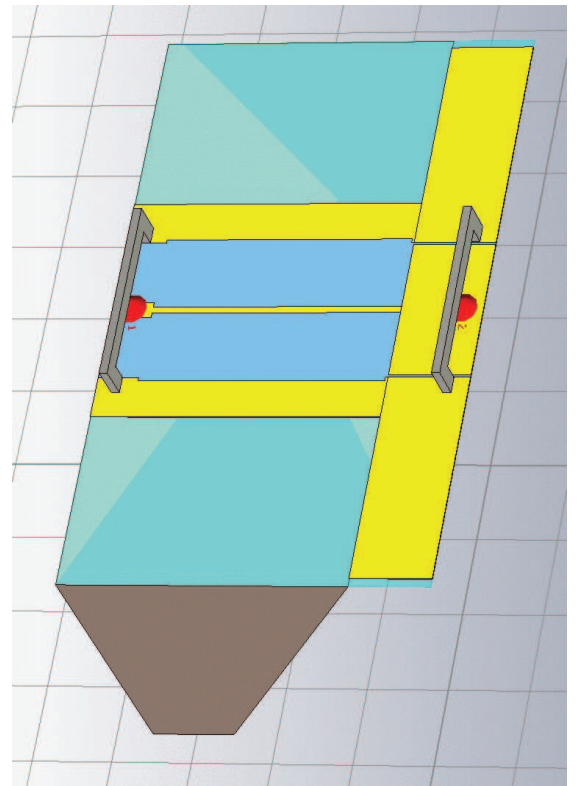

Fig. 7. CST model of silicon-to-membrane transition.

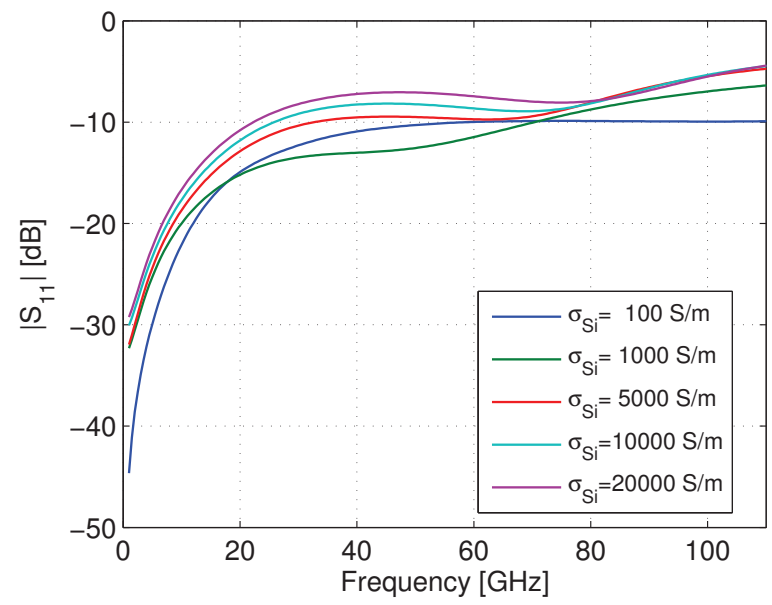

Fig. 8. Simulated reflection of silicon-to-membrane transition at port 1 , with variation of silicon substrate conductivity $\sigma_{\mathrm{Si}}$.

1993) for substrate conductivities above $5000 \mathrm{~S} \mathrm{~m}^{-1}$. Above $80 \mathrm{GHz}$ (not shown here), the measured results become very noisy. This is due to the fact that the reference calibration standards on the GaAs substrate were not designed for operation at such high frequencies. In (Rumiantsev et al., 2009), where also alternative schemes for determining $Z_{0}$ were discussed, similar observations were made.

\subsection{Numerical simulations}

Numerical simulations of the test structures were performed using the 3-D full-wave time-domain electromagnetic field solver Microwave Studio (2011) as mentioned before. Fig-

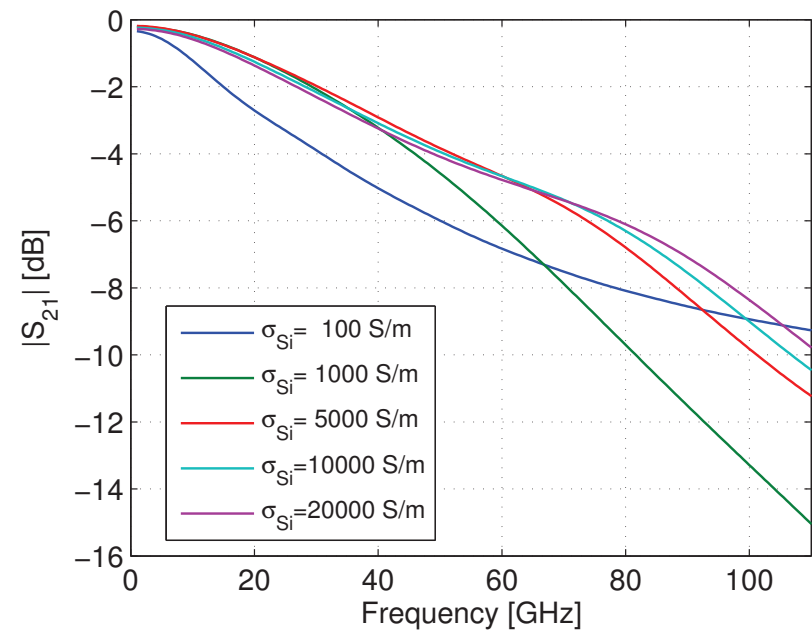

Fig. 9. Simulated transmission of silicon-to-membrane transition, with variation of silicon substrate conductivity $\sigma_{\mathrm{Si}}$.

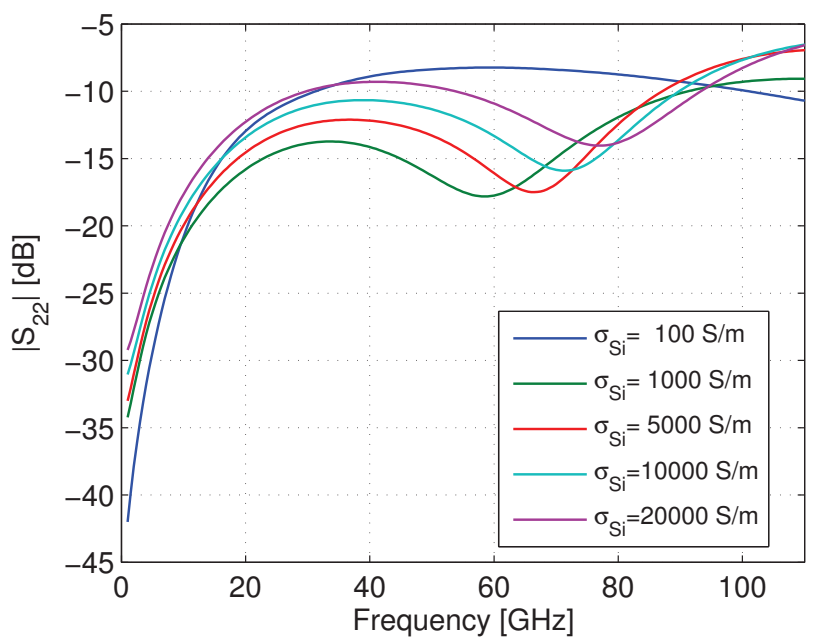

Fig. 10. Simulated reflection of silicon-to-membrane transition at port 2 , with variation of silicon substrate conductivity $\sigma_{\mathrm{Si}}$.

ure 7 shows the port description used in the electromagnetic simulation. Instead of modeling the microwave probe, we used a conducting bridge between the ground planes as a substitute for a much more complicated probe model. This port description is similar to the PEC ground bridge used for HFSS simulations in (Johansen et al., 2007). Port 1 is located at the contact pads, port 2 is located $75 \mu \mathrm{m}$ inside the membrane CPW.

The simulated scattering parameters of the silicon-tomembrane transition, with variation of the substrate conductivity $\sigma_{\mathrm{Si}}$, are shown in Figs. 8-10. The results for both reflection and transmission do not vary much over frequency for conductivities above $5000 \mathrm{~S} \mathrm{~m}^{-1}$. The reflection coefficients stay below $-10 \mathrm{~dB}$ for a wide frequency range. 


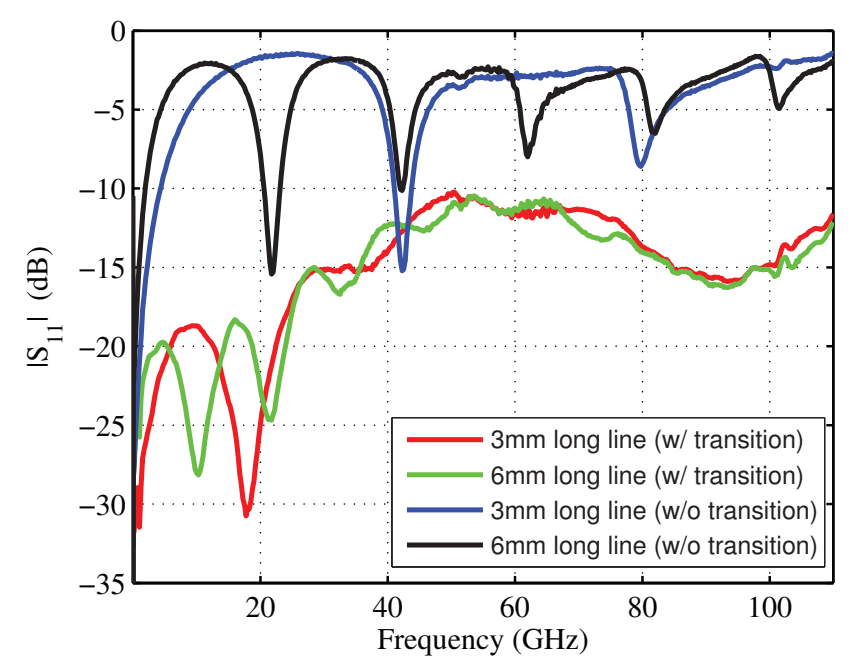

Fig. 11. Measured reflection coefficient of $3 \mathrm{~mm}$ and $6 \mathrm{~mm}$ long membrane CPWs with and without transitions.

We also performed parametric studies of the geometrical cross section of the silicon access lines to confirm the final geometry chosen for the layout of the interconnect structures (not shown here).

\subsection{Measured transition performance}

To investigate the broadband performance of the silicon-tomembrane transitions, the membrane CPWs were produced both with and without transitions at either side of the lines. The ones without transitions were configured with an additional length of $50 \mu \mathrm{m}$ on the silicon substrate to allow for on-wafer probing.

The measured reflections of a $3 \mathrm{~mm}$ and $6 \mathrm{~mm}$ long membrane CPW with (w/) and without (w/o) transitions are compared in Fig. 11. The raw S-parameter measurements were corrected with the aid of an off-wafer GaAs multiline TRL calibration whose reference impedance was set to $50 \Omega$. Figure 11 demonstrates that the reflection properties greatly improve when using the transitions, independent of the membrane CPW length.

\section{Membrane CPW characterization}

To characterize the membrane CPW, its broadband properties were determined in the frequency range of $1-110 \mathrm{GHz}$ applying the model verified in (Arz et al., 2011a). The membrane CPW's cross-sectional parameter values are given in Table 3. Comparing the calculated propagation constant against the propagation constant extracted using multiline TRL (Marks, 1991) verified the results. One offset short and four lines with lengths between $0.5 \mathrm{~mm}$ and $7.072 \mathrm{~mm}$ were applied for the multiline TRL calibration. All test structures contained broadband transitions as discussed in Sect. 2. The vector
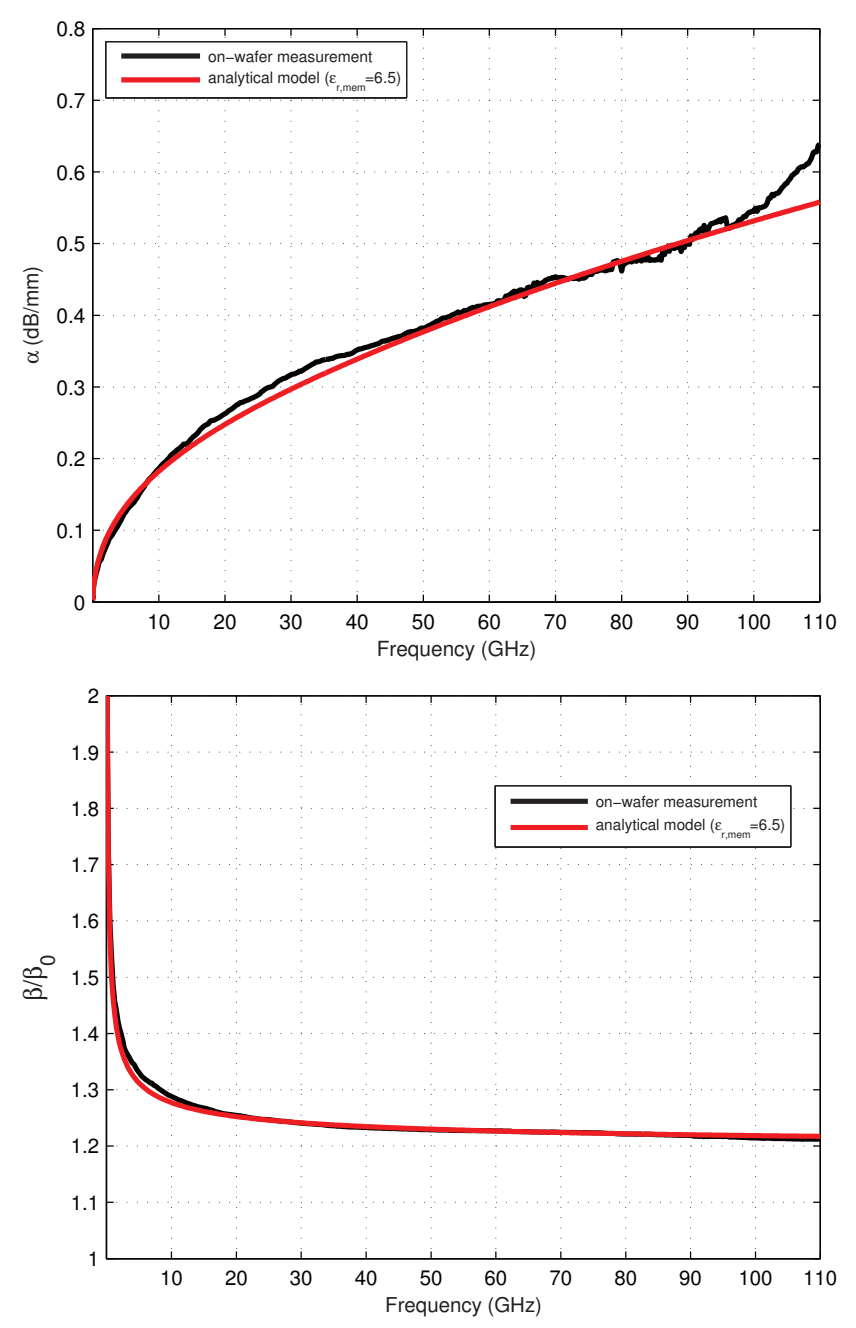

Fig. 12. Attenuation constant $\alpha$ and normalized phase constant $\beta /$ $\beta_{0}$ of membrane CPW.

Table 3. Membrane CPW parameters.

\begin{tabular}{rr}
\hline parameter & value \\
\hline$w_{g}$ & $263 \mu \mathrm{m}$ \\
$w$ & $172 \mu \mathrm{m}$ \\
$s$ & $3 \mu \mathrm{m}$ \\
$t$ & $0.9 \mu \mathrm{m}$ \\
$\sigma_{\text {metal }}$ & $30 \mathrm{MS} \mathrm{m}^{-1}$ \\
$\varepsilon_{\mathrm{r}, \mathrm{mem}}$ & 6.5 \\
$t_{\text {mem }}$ & $1 \mu \mathrm{m}$ \\
$\tan \delta$ & 0.0001 \\
\hline
\end{tabular}

network analyzer Anritsu MS4647A was employed for the on-wafer scattering parameter measurements of the line and reflect standards.

Figure 12 shows the measured and calculated attenuation constant $\alpha$ and the phase constant $\beta$ normalized to the free-space value $\beta_{0}$ for the membrane CPW according to 
Table 3. The agreement between measurement and calculation is good for $\alpha$, and excellent for $\beta / \beta_{0}$ in the entire frequency range.

Further investigations presented in (Arz et al., 2011b) indicate a tangible sensitivity of $\alpha$ and, even more, $\beta$, to the relative permittivity of the membrane material $\varepsilon_{\mathrm{r}, \mathrm{mem}}$. Despite the fact that the thickness of the membrane layer is just $1 \mu \mathrm{m}$, the sensitivity is very high. Together with the sensitivity of $Z_{0}$ in the silicon access lines, this may open up an opportunity to determine the broadband relative permittivity of thin-films from on-wafer measurements.

\section{Conclusions}

The new interconnect structure presented in this paper is suitable for on-wafer probing on highly-conductive silicon substrates up to at least $110 \mathrm{GHz}$. A reflection coefficient less than $-10 \mathrm{~dB}$ was measured in the complete frequency region. Both the silicon and the membrane technology part of this interconnect structure were characterized individually by measurement and calculation.

The silicon-to-membrane transition allows to accurately deembed the broadband electromagnetic properties of the membrane CPW part of the interconnect structure. Measured and calculated values of the propagation constant of the membrane CPW agree well in the entire frequency range. Possible applications of the new interconnect structures include, but are not limited, to wideband high-precision onwafer calibration standards.

Acknowledgements. The authors would like to thank the Test and Measurement Division, Rohde\&Schwarz GmbH \& Co. KG, München, Germany, for providing the membrane material, and Dipl.-Ing. (FH) Dirk Schubert from PTB, Germany, for performing the on-wafer measurements. One of the authors (Stephanus Büttgenbach) gratefully acknowledges the financial support of the Volkswagen Foundation.

\section{References}

Arz, U., Williams, D. F., and Grabinski, H.: Characteristic impedance measurement on silicon, in: Interconnects in VLSI Design, Grabinski, H., Kluwer Academic Publishers, 147-154, 2000.

Arz, U., Rohland, M., Kuhlmann, K., and Büttgenbach, S.: Wideband Electromagnetic Modeling of Coplanar Waveguides Fabricated in Membrane Technology, in: 15th IEEE Workshop on Signal Propagation on Interconnects, Naples, Italy, 8-11 May 2011, 129-130, doi:10.1109/SPI.2011.5898856, $2011 \mathrm{a}$.

Arz, U., Rohland, M., Kuhlmann, K., and Büttgenbach, S.: Optimized Coplanar Waveguides in Membrane Technology for Wideband On-Wafer Calibrations, in: 20th IEEE Conference on Electrical Performance of Electronic Packaging and Systems, San Jose, California, 23-26 October, 77-80, doi:10.1109/EPEPS.2011.6100191, 2011b.

Grotelüschen, E., Dutta, L. S., and Zaage, S.: Full-wave analysis and analytical formulas for the line parameters of transmission lines in semiconductor substrate, Integration, the VLSI journal, 16, 33-58, doi:10.1016/0167-9260(93)90057-J, 1993.

Heinrich, W.: Quasi-TEM description of MMIC coplanar lines including conductor-loss effects, IEEE T. Microw. Theory., 41, 4552, doi:10.1109/22.210228, 1993.

Johansen, T. K., Jiang, C., Hadziabdic, D., and Krozer, V.: EM Simulation Accuracy Enhancement for Broadband Modeling of On-Wafer Passive Components, in: 37th IEEE European Microwave Conference, Munich, Germany, 8-12 October, 12451248, doi:10.1109/EUMC.2007.4405426, 2007.

Marks, R. B.: A multiline-method of network analyzer calibration, IEEE T. Microw. Theory., 39, 1205-1215, doi:10.1109/22.85388, 1991.

Microwave Studio, CST, Darmstadt, Germany, 2011.

Rohland, M., Arz, U. and Büttgenbach, S.: Benefits of on-wafer calibration standards fabricated in membrane technology, Adv. Radio Sci., 9, 19-26, doi:10.5194/ars-9-19-2011, 2011.

Rumiantsev, A., Corson, P., Sweeney, S., and Arz, U.: Applying the Calibration Comparison Technique for Verification of Transmission Line Standards on Silicon up to $110 \mathrm{GHz}$, in: 73rd ARFTG Microwave Measurement Conference, Boston, MA, 12 June 2009, 67-72,doi:10.1109/ARFTG.2009.5278064, 2009. 